II.

\title{
Die Entstehung und das Wesen der baconischen Methode.
}

Von

\section{Giuseppe Furlani.}

i

(SchluB.)

Bevor wir auf eine nähere Besprechung eingehen, wollen wir hier den englischen Text des 11. Kapitels wiedergeben, da die große BaconAusgabe von Ellis, Spedding und Heath nicht gerade zu den oft vorkommenden und allgemein zugänglichen Büchern gehört, und eine deutsche Übersetzung folgen lassen, um so das Verständnis zu erleichtern und eine Kontrolle unserer Auffassung zu ermöglichen. Unsere Übersetzung ist nicht immer wörtlich, da eine solche notwendigerweise noch dunkler ausgefallen wäre als das englische Original selbst. Hie und da haben wir frei paraphrasiert und nicht den genauen Wortlaut, sondern nur den Sinn so entsprechend wie möglich wiedergegeben. Der Übersicht und der Zitate halber haben wir die einzelnen Absätze numeriert. Im Original ist das ganze Kapitel fortlaufend geschrieben.

1. It appeareth then what is now in proposition not by general circumlocution but by particular note. No former philosophy varied in terms or method; no new placet or speculation upon particulas already known; no referring to action by any manual of practice; but the revealing and discovering of new inventions and operations. 2. This is to be done without the errors and conjectures of art, or the length and difficulties of experience; the nature and kinds of which inventions have been described as they could be discovered; for your eye cannot pass one kenning without further sailing; only we have stood upon the best advantaces of the notions received, as upon a mount, to shew the knowledges adjacent and confining. 3 . If therefore the true and of knowledge not propounded hath bred large error, the best and perfectest condition of the same end not perceived will cause some declination. 4. For when the butt is set up men need not rove, but except the white be placed men cannot level. This perfection we 
mean not in the worth of the effect, but in the nature of the directiom; for our purpose is not to stir up men's hopes, but to guide their travelss. 5 . The fulness of direction to work and produce any effect consistetih in two conditions, certainty and liberty. 6. Certainty is when the direction is not only true for the most part, but infallible. 7. Liberty is when the direction is not restrained to some definite means, but comprehendeth all the means and ways possible; for the poet saith well: Sapientibus undique latae sunt viae, and where there is the greatest plurality of change, there is the greatest singularity of choicce. 8. Besides as a conjectural direction maketh a casual effect, so a particular and restrained direction is no less casual than an uncertain. 9. For those particular means whereunto it is tied may be out of your power or may be accompanied with an overvalue of prejudice; and so if for want of certainty in direction you are frustrated in success, for want of variety in direction you are stopped in attempt. 10. If therefore your direction be certain, it must refer you and point you to somewhat, which if it be present; the effect you seek will of necessity follow, else may you perform and not obtain. 11. If it be free, then must it refer you to somewhat which if it be absent the effect you seek will of necessity withdraw, else may you have power and not attempt. 12. This notion Aristotle had in light, though not in use. For the two commended rules by him set down, whereby the axioms of scienees are precepted to be made convertible, and which the latter men have not without elegancy surnamed the one the rule of truth because it preventeth deceit, the other the rule of prudence because it freeth election, are the same thing in speculation and affirmation which we now observe. 13. An example will make my meaning attained, and yet percase make it thought that they attainet it not. 14. Let the effect to be produced be Whiteness; let the first direction be that if air and water be intermingled or broken in small portions together, whiteness will ensue, as in snow, in the breaking of the sea and rivers, and the like." 15 . This direction is certain, but very particular and restrained, being tied but to air and water. 16. Let the second direction be, that if air be mingled as before with any transparent body, such nevertheless as is uncoloured and more grossly transparent than air itself, that then, as glass or crystal, \&c. being beaten to fine powder, by the interposition of the air becometh white; the white of an egg being clear of itself, receiving air by agitation becometh white, receiving air by concoction becometh white; here 
\$ou are freed from water, and advanced to or clear body, and still tied to air. 17. Let the third direction exclude or remove the restraint of an uncoloured body; as in amber, sapphires Etc., which beaten to fine powder become white; in vine and beer, which brought to froth become white. 18. Let the fourth direction exclude the restraint of a body more grossly transparent than air, as in flame, being a body compounded between air and a finer substance than air; which flame, if it were not for the smoke, which is the third substance which incorporateth itself and dyeth the flame, would be more perfect white. 19 . In all these four directions air still beareth a part. 20 . Let the fifth direction then be, that if any bodies, both transparent but in an unequal degree, be mingled as before, whiteness will follow, as oil and water beaten to an ointment, though by settling the air which gathereth in the agitation be evaporate, yet remaineth white; and the powder of glass or crystal put into water, whereby the air giveth place, yet remaineth white, though not so perfect. 21. Now are you freed from air, but still you are tied to transparent bodies. 22. To ascend further by scale I do forbear, partly because it would draw on the example to an over great length, but chiefly because it would open that which in this work I determine to reserve; for to pass through the whole history and observation of colours and objects visible were to long a digression; and our purpose is now to give an example of a free direction, thereby to distinguish and describe it; and not to set down a form of interpretation how to recover and attain it. 23. But as we intend not now to reveal, so we are circumspect not to mislead; and therefore (this warning being given) returning to our purpose in hand, we admit the sixth direction to be, that all bodies or parts of bodies which are unequal equally, that is in a simple proportion, do represent whiteness; we will explain this, though we induce it not. 24 . It is then to be understood, that absolute equality produceth transparence, inequality in simple order or proportion produceth whiteness, inequality in compound or respective arder or proportion produceth all other colours, and absolute or orderless inequality produceth blackness; which diversity, if so gross a demonstration be needful, may be signified by four tables; a blank, $\checkmark$ chequer, a fret, and a medley; whereof the fret is evidént to admit creat variety. 25. Out of this assertion are satisfied a multitude of ffects and observations, as that whiteness and blackness are most ncompatible with transparence; that whiteness keepeth. light and 
blackness stoppeth light, but neither passeth it; that whiteness and blackness are never produced in rainbows, diamonds, crystals, and the like; that white giveth no dye, and black hardly taketh dye; that whiteness scemeth to have an affinity with dryness, and blackness with moisture; that adustion causeth blackness, and calcination whitteness; that flowers are generally of fresh colours, and rarely black ettc. 26. All which I do now mention confusedly by way of derivation and not by way of induction. 27. This sixth direction, which I have thus explained, is of good and competent liberty for whiteness fixed and inherent, but not for whiteness fantastical or appearing, as shall be afterwards touched. 28. But first do you need a reduction back to certainty or verity; for it is not all position or contexture of unequal bodies that will produce colour; for aqua fortis, oil of vitriol, etc. more manifestly, and many other substances more obscurely, do consist of very unequal parts, which yet are transparent and clear. 29. Therefore the reduction must be, that the bodies or parts of bodies so intermingled as before be of a certain grossness or magnitude; for the unequalities which move the sight must have a further dimension and quantity. than those which operate many other effects. 30. Some few grains of saffron will give a tincture to a tun of water; but so many grains of civet will give a perfume to a whole chamber of air. 31. And therefore when Democritus (from whom Epicurus dire borrow it) held that the position of the solid portions was the cause of colours, yet in the very truth of his assertion he should have added, that the portions are required to be of some magnitude. 32. And this is one cause why colours have little inwardness and necessitude with the nature and proprieties of things, those things resembling in colour which otherwise differ most sas salt and sugar, and contrariwise differing in colour which otherwise resemble most, as the white and blue violets, and the several veins of one agate or marble, by reason that other virtues consist in more subtile proportions than colours do, and yet are there virtues and natures which require a grosser magnitude than colours, as well as scents and divers others require a more subtile; for as the portion of a body will give forth scent which is to small to be seen, so the portion of a body will shew colours which is to small to be endued with weight; and therefore one of the prophets with great elegancy describing how all creatures carry no proportion towards God the creator, saith, That all the nations in respect of 
hihim are like the dust upon the balance, which is a thing appeareth bbut weigheth not. 33. But to return, there resteth a further freeing of t]this sixth direction; for the clearness of a river or stream sheweth wwhite at a distance, and crystalline glasses deliver the face or any 0 other object falsified in whiteness, and long beholding the snow to a a weak eye giveth an impression of azure rather than of whiteness. 334. So as for whiteness in apparition only and representation by the qqualifying of the light, altering the intermedium, or affecting the eye iitself, it reacheth not. 35. But you must free your direction to the pproducing of such an incidence, impression, or operation, as may cause a a precise and determinate passion of the eye; a matter which is much nmore easy to induce than that which we have passed through; but yyet because it hath a full coherence both with that act of radiation ('which hath hitherto been conceived and termed so unproperly and uuntruly by some an effluxion of spiritual species and by others an iinvesting of the intermedium with a motion which successively is cconveyed to the eye) and with the act of sense, wherein I should lilikewise open that which I think good to withdraw, I will omit. 36. Neither do I contend but that this motion which I call the freeing oof a direction, in the received philosophies (as far a swimming anticcipation could take hold) might be perceived and discerned; being not nmuch other matter than that which they did not only aim at in the ttwo rules of Axioms before remembered, but more nearly also in that which they term the form or formal cause, or that which they call tthe true difference; both which nevertheless it seemeth they propound rrather as impossibilities and wishes than as things within the compass oof human comprehension. 37. For Plato casteth his burden and saith that he will revere him as a God, that can truly divide and define; which ccannot be but by true forms and differences. 38 . Wherein I join hands wwith him, confessing as much as yet assuming to myself little; for if any nman can by the strength of his anticipations find out forms, I will rmagnify him with the foremost. 39. But as any of them would say that if divers things which many men know by instruction and cobservation another knew by revelation and without those means, tthey would take him for somewhat supernatural and divine; so I do aacknowledge that if any man can by anticipations reach to that which aa weak and inferior wit may attain to by interpretation, he cannot rreceive too high a title. 40. Nay I for my part do indeed admire to 
see how far some of them have proceeded by their anticipations; but how? it is as I wonder at some blind men, to see what shift they make without their eye-sight; thinking with myself that if I were blind I could hardly do it. 41. Again Aristotle's school confesseth that there is no true knowledge but by causes, no true cause but the form, no true form known except one, which they are pleased to allow; and therefore thus far their evidence standetb with us, that both hitherto there hath been nothing but a shadow of knowledge, and that we propound now that which is agreed to be worthiest to be sought, and hardest to be found. '42. There wanteth now a part very necessary, not by way of supply but by way of caution; for as it is seen for the most part that the outward tokens and badges of excelleney and perfection are more incident to things merely counterfeit than to that which is true, but for a meaner and baser sort; as a dubline is more like a perfect ruby than a spinel, and a counterfeit angel is made more like a true angel than if it were an angel coined of China gold; in like manner the direction carrieth a resemblance of a true direction in verity and liberty which indeed is no direction at all. 43. For though your direction seem to be certain and free by pointing you to a nature that is unseparable from the nature you inquire upon, yet if it do not carry you on a degree or remove nearer to action, operation, or light to make or produce, it is but superficial and counterfeit. 44. Wherefore to secure and warrant what is a true direction, though that general note I have given be perspicuous in itself (for a man shall soon cast with himself whether he be ever the nearer to effect and operate or no, or whether he have won but an abstract or varied notion) yet for better instruction I will deliver three particular. notès of caution. 45. The first is that the nature discovered be more original than the nature supposed, and not more secondary or of the like degree; as to make a stone bright or to make it smooth it is a good direction to say, make it even; but to make a stone even it is no good direction to say, make it bright or make it smooth; for the rule is that the disposition of any thing referring to the state of it in itself or the parts, is more original than that which is relative or transitivo towards another thing. 46. So evenness is the disposition of the stone in itself, but smooth is to the hand and bright to the eye, and yet nevertheless they all cluster and concur; and yet the direction is more urperfect, if it do appoint you to such a relative as is in the same kind 
and not in a diverse. 47. For in the direction to produce brightness by smoothness, although properly it win no degree, and will never teach you any new particulars before unknown; yet by way of suggestion or bringing to mind it may draw your consideration to some particulars known but not remembered; as you shall sooner remember some practical means of making smoothness, than if you had fixed your consideration only upon brightness; but if the direction had been to make brightneass by making reflexion, as thus, make it such as you may see your face in it, this is merely secondary, and helpeth neither by way of informing nor by way of suggestion. 48. So if in the inquir.y of whiteness you were directed to make such a colour as should be seen furthest in a dark light; here you are advanced nothing at all. 49. For these kinds of natures are but proprieties, effects, circumstances, concurrences, or what else you shall like to call them, and not radical and formative natures towards the nature supposed. 50. The second caution is that the nature inquired be collected by division before composition, or to speak more properly, by composition subaltern before you ascend to compositir,n absolute ete.

\section{Übersetzung.}

Es erhellt, daß das, worum es sich jetzt handelt, nicht im allgemeinen, sondern im besonderen behandelt werden muB. Von den früheren Philosophien waren alle, den Begriffen und der Methode nach, gleich. Keine neuen Theorien und Spekulationen über das Besondere waren bekannt; kein Handbuch der Praxis nahm auf das Handeln Rücksicht; dagegen ist nun unsere Aufgabe die, neue Erfindungen und Werke zu enthüllen und zu entdecken. Das muß ohne die Irrtümer, welchen die bloße Kunstfertigkeit ausgesetzt ist und ohne die Langwierigkeit und Schwierigkeit der vulgären Erfahrung ausgeführt werden. Das Wesen und die Arten jener Erfindungen sind als erfindbare beschrieben worden. Denn das Auge kann nicht eine Zielscheibe übersehen, ohne weiter zu schweifen; bis jetzt ist die Menschheit bei den bestmöglichen Vorteilen, die die althergebrachten Begriffe verschafften, stehen geblieben, gleichsam auf einem Berge, nur die anliegenden und angrenzenden Wissensfelder zu überschauen. Wenn deshalb der Umstand, daß man nicht den Zweck der Erkenntnis im Auge hatte, viele Irrtümer erzeugte, so wird der Umstand, daß man 
den echten Zweck nicht erkennt, eine Abweichung vom richtigen Wege verursachen. Denn ist die Zielscheibe aufgestellt, so braucht man nicht mit dem Blicke umherzuschweifen, aber ist der Zielpunkt der Scheibe nicht da, so kann man nicht visieren. Diese Vollkommenheit der Handlung des Menschen soll nicht unserer Meinung nach in dem hohen Werte der durch die Handlung zu erzielenden Wirkung besteh en, sondern in dem Wesen und der Art der ,Richturng"; denn es ist nicht unsere Absicht, nur leere Hoffnungen zu-entfachen, sondern die Erfindungsreisen des Menschen zu leiten. Die „Fülle" der „Richtung" für das Handeln und Hervorbringen irgend einer Wirkung besteht in zwei Bedingungen: Gewißheit und Freiheit. Gewißheit der ,Richtung" liegt dann vor, wenn die „Richtung“ nicht nur für einige Fälle wahr ist, sondern immer unfehlbar ist. Freiheit der "Richtung" besteht dann, wenn die „Richtung" nicht auf einige bestimmte Mittel zur Erzeugung der angestrebten Wirkung beschränkt ist, sondern alle dafür möglichen Mittel und Wege umfaßt; denn sehr gut sagt der Dichter: Sapientibus undique latae sunt viae; denn wo es die größte Anzahl von Änderungsmöglichkeiten der Erzeugungsmittel gibt, dort ist auch die größte Besonderheit in der Wahl eines dieser Mittel möglich. Überdies: wie eine. hypothetische „Richtung“ eine nur zufällige Wirkung hervorbringt, so ist auch eine partikuläre und beschränkte Richtung nicht weniger - was den Erfolg der Handlung betrifft zufällig, als eine unsichere. Denn die nur besonderen Mittel, die bei einer partikulären „Richtung“" dem Menschen zur Verfügung stehen kömnen und an welche eben die ,Richtung ${ }^{\text {"a }}$ gebunden ist; können ja. gerade außerhalb unserer Machtsphäre liegen oder doch ihre Beschaffung oder Anwendung mit großen Schwierigkeiten oder Schäden und Verlusten verbunden sein; und so kommt es, daß, wenn man aus Mangel an Gewißheit der „Richtung“" des Erfolges der Hansllung beraubt ist, man aus Mangel an Verschiedenheit der ,Richtung" in seinen Versuchen neue "Richtungen" zu entdecken, aufgehalten wird. Wenn also die „Richtung" gewiß sein soll, so muß sie uns hinweisen und. hinrichten auf ein Etwas, auf das, wenn es da ist, die angestrebte Wirkung notwendigerweise folgt, denn sonst handeln wir zwar, erreichen aber die gesuchte Wirkung nicht. Ist die „,Richtung" dagegen frei, so weist sie uns auf Etwas, bei dessen Fehlen notwendigerweise auch die gesuchte Wirkung verschwindet, denn sonst könnten wir die Macht haben, aber nicht versuchen. Dieser Begriff der , Riçl- 
tuung"' schwebte dem Aristoteles zwar in Theorie vor, aber er gebrauchte ihmn nicht in der Praxis. Denn die beiden oben genannten, von ihm auufgestellten Regeln, durch welche die Axiome der Wissenschaften umkeehrbar gemacht werden sollen und welche die Neueren in nicht ineleganter Weise die „Regel der Wahrheit“, weil sie uns vor dem Irrtum beehütet, und die „Regel der Klugheit" genannt haben, weil sie die WWahl des-Erzeugungsmittels einer Wirkung frei macht, sind auf dem Gưebiete der Spekulation und der theoretischen Behauptungen ebenddasselbe, was wir, jetzt auf dem Gebiete der Praxis, des Handelns boefolgen wollen. Ein Beispiel möge das; was ich meine, darlegen; unnd doch wird gerade dieses Beispiel vielleicht den Anschein erwecken, $\mathrm{dda} B$ die aufgestellten Regeln das, was ich meinè, nicht getroffen haben. D)ie hervorzubringende Wirkung solle die Weiße sein; es möge die errste Richtung folgendermaßen lauten: wenn Luft und Wasser mit eiinander gemischt oder in kleine Teile untereinander zerstïckelt wwerden, so wird darauf Weiße folgen; wie z. B. im Falle des Schnees, ooder wenn das Meer oder Flüsse ibre Wellen an den Ufern brechen uund in ähnlichen Fällen. Eine solche „Richtung" ist gewiß, aber sehr paartikulär und beschränkt, da sie an Wasser und Luft gebunden ist. Die zweite „Richtung" möge so lauten: Wenn Luft mit irgend einem ddurchsichtigen Körper in der schon früher angegebenen Weise, aber mit eæinem solchen durchsichtigen Körper, der ungefärbt ist und. weniger durchsichtig als die Luft selbst ist (z. B. Glas oder Kristall) vermischt nwird, das heißt, wenn ein solcher Körper zu feinem Pulver zerschlagen wird so wird es dureh dàs Dazwischentreten der Luft zwischen die einzehen feinen Teilchen weiß; z. B. das Weiße des Eies, das an und für siêt durchsichtig ist, wird weib, wenn es durch,Rührung oder ddurch Aussottung Luft erhält. In diesem Falle sind wir zwar das Wasser los geworden und zu einem durchsichtigen Körper fortgreschritten, aber noch immer an die Luft gebunden. Möge die dritte Richtung den Umstand - der einer Beschränkung gleich kommt -, dlaß es sich um einen f arblos en Körper handelt, ausschließen. Z. B. Hmbra, Saphyr zu feinem Pulver zerschlagen, werden weiß, oder Wein und Bier zu Schaum geschlagen, werden ebenfalls weiß: Möge die wierte „Richtung" den weniger als Luft durchsichtigen Körper aussschließen, wie es z. B. bei der Flamme der Fall ist, die ein aus Luft und einem feineren Körper als Luft zusammengesetzter Körper ist; sie würde, wenn nicht auch der Rauch da wäre, der als dritte Substanz 
mit ihr sich vorbindet und sie färbt, vollkommen weiß sein. In allen diesen vier "Richtunger" spielt aber die Luft noch eine Rolle. Es möge die fünfte „Richtung" folgende sein: wenn irgendwelche zwei durchsichtige, aber in verschiedenem Grade durchsichtige Körper in der angegebenen Weise zusammen vermischt werden, so wird die Weiße da sein, so z. B. wenn Öl und Wasser in ungleichem Verhältnis zusammengemischt werden, so bleibt das Gemisch dennoch weiß, wenn man auch die Luft, die durch das Schütteln sich noch darin befindet, verdampfen läßt; und Glas- oder Kristallstaub in Wasser geworfen - wodurch die Luft entweicht - bleibt dennoch weiß, obwohl nicht ganz vollkommen weiß. Jetzt sind wir zwar die Luft los geworden, aber sind noch immer an durchsichtige Körper gebunden. Ich will nicht weiter von Stufe zu Stufe fortschreiten, zum Teil, weil dies mein Beispiel zu sehr in die Länge ziehen würde, aber hauptsächlich darum, weil es das ans Tageslicht ziehen würde, womit ich in diesem Werke noch zurückhalten will; denn die ganze Geschichte und Beobachtung über die Farben und die sichtbaren Gegenstände durchzugehen, wäre ein zu großer Abstecher, während es jetzt unsere Absicht ist, nur ein Beispiel einer freien ,Richtung"s zu geben und sie dadurch zu beschreiben und abzugrenzen, und nicht die Art des Verfahrens anzugeben, wie man sie erlangen und erreichen kann. Da wir aher jetzt nituis verraten wollen, so hüten wir uns auch davor, den Leser in die Irre zu führen. Deshalb kehren wir, nachdem wir diese Warnung gemacht haben, zu unserem Thema zurück und wollen annehmen, daß die sechste „Richtung" so laute: Alle Körper oder Teile von Körpern, welche in einfachem Verhältnisse zu einander stehen, weisen Weiße aufi ${ }^{9}$ ); wir wollen dies erklären, obwohl wir es nicht induziert haben. Darunter ist also zu verstehen, daß absolute Proportionalität zwischen den Körperteilen Durchsichtigkeit erzeugt, einfache Ungleichheit erzeugt Weiße, zusammengesetzte Ungleichheit erzeugt alle die übrigen Farben und absolute Ungleichheit erzeugt Schwärze; diese Verschiedenheit kann - wenn ein so gewichtiger Beweis nötig wäre - durch vier Tafeln dargestellt werden, durch eine lẹere, eine scheckige, eine gestreifte und eine bunte, wovon die ge-

9) In ähnlicher Fassung kehrt diese, ,Richtung" in De Augm. III. 4(I. 506) wieder: At in Metaphysica si fiat inquisitio hujusmodi quidpiam reperies: Gorpora duo Diaphana intermixta Portionibus corum Opticis simplici crdine sive aequaliter collocatis, constituere Albedinem. 
streifte Tafel einleuchtenderweise eine große Abwechslung zuläßt. Durch diese unsere Behauptung leistet man einer Menge von Wirkungen und Beobachtungen Genüge, wie z. B. daß Weiße und Schwärze ganz unvereinbar mit Durchsichtigkeit sind, daß Weiße das Licht behält und Schwärze es aufhält, aber es nicht durchläßt, daß Weiße und Schwärze nie in Regenbogen, Diamanten, Kristallen und ähnlichem entsteht; daß Weiß keine Farbe gibt und Schwarz nur schwer sich färben läßt; daß die Weiße mit der Trockenheit eine gewisse Verwandtschaft zu haben scheint und die Schwärze mit der Feuchtigkeit; daß Verbrennung Sčhwärze hervorruft und Verkalkung Weiße; daß die Blumen im allgemeinen frischfarbig sind und selten schwarz usw. All dies erwähne ich dureheinander auf dem Wege der Ableitung und nicht auf dem der Induktion. Die eben erklärte sechste „Richtung“ besitzt Freiheit, die gut und angemessen ist für feststehende und inhärente Weiße, nicht aber für die Weiße, welche eine bloße Vorstellung und Erscheinung ist, wie ich später auseinandersetzen werde. Aber zuerst ist da eine Zurüekführung der „Richtung" auf Gewißheit oder Wahrheit nötig; denn nicht jede Lage oder Vermischung von ungleichen Körpern bringt die Farbe hervor; denn Königswasser, - Vitriolöl und so weiter in ganz offenbarer Weise und viele andere Körper in mehr dunkler Weise, bestehen aus sehr ungleichartigen Teilen, sind aber dennoch durchsichtig und hell. Deshalb muß die Zurückführung jener "Richtung“ folgendermaßen lauten: die in der angegebenen Weise vermischten Körper oder Teile von Körpern müssen eine bestimmte Größe haben, denn die Ungleichbeiten derselben, welche unsere Gesichtssinne affizieren, müssen eine größere Ausdehnung und Stärke haben als diejenigen, welche andere Wirkungen erzeugen. Wenige Saffran-Körner färben eine Tonne Wassers, aber ebensoviele Körner Zibet verleihen der Luft eines ganzen Zimmers ihren Duft. Und deshalb hat auch Demokrit (von dem es Epikurus entlehnte) behauptet, daß die Lage der festen Teile der Körper die Ursache der Furben ist, so hätte er doch, damit seine Behauptung vollkommen wahr sei, noch hinzufügen müssen, daß jene Teile eine bestimmte Größe besitzen müssen. Das ist die Ursache, weshalb die Farben wenig Irwendigkeit in bezug auf die Körper, denen sie anhaften, haben und nicht in notwendigem Zusammenhange mit dem Wesen und den wasenhaften Eigenschaften der Körper stehen, da ja Dinge, welche der Farbe nach ähnlich sind, in anderen Beziehungen große Unter- 
schiede aufweisen (Salz - Zucker), und umgekehrt der Farbe narch verschieden sind und in anderen Beziehungen ähnlich sind (weiße und blaue Veilchen, die verschiedenen Adern eines und desselben Agatsteines oder Marmorstückes); und dies aus dem Grunde, weil andere Naturen als die Farben in feineren Verhältnissen als die Farben bestehen; und doch gibt es Eigenschaften und Naturen, die eine größere Größe der Körperteile erfordern als die Farben, ebenso wie Gerüche und andere Naturen eine geringere nötig haben; denn ebenso wie die Teile eines Körpers einen Geruch von sich geben, die zu klein sind als daß sie gesehen werden könnten, so können auch die Teile eires Körpers eine Farbe aufweisen, díe zu klein sind, um ein Gewicht zu haben. Und deshalb hat einer der Propheten mit großer Eleganz bei der Beschreibung der Inkommensurabilität der Kreaturen mit Gott, dem Schöpfer, gesagt, daß alle Völker Gott gegenüber wie Staub auf der Wagschale sind, denn Staub ist ein Ding, das gesehen wird, aber kein Gewicht hat. Aber, um zu unserem Thema zurückzukehren: es bleibt uns noch übrig unsere'sechste „Richtung" noch weiter frei $\mathrm{zu}$ machen; denn die Helligkeit eines Stromes zeigt bei einer gewissen Entfernung Weiße, und Krystall-Gläser spiegeln unser Antlitz oder irgend einen anderen Gegenstand mit Weiße verfälscht wieder, und durch längere Zeit vor ein schwaches Auge gehaltener Schnee gibt mehr die Empfindung der blauen als der weißen Farbe. So daß die von uns aufgestellte letzte „Richtung" die Erzeugung der Weiße; die im Falle, daß es sich um Weiße, die bloß eine Erscheinung und rein subjektive Vorstellung ist, handelt, mittels Änderung des Mediums oder durch eine Einwirkung auf das Auge erzielt wird, nicht gewährleistet. Sondern man muß eben diese letzte "Richtung" noch freier ausgestalten bis zur Hervorbringung eines solchen Einfallswinkels des Lichtes oder einer Beeinflussung des Auges, die eine bestimmte Wirkung im Auge erzeugt (nämlich die Weiße); dies ist aber leichter zu induzieren als das, was wir früher besprochen haben; aber da es in inniger Beziehung steht zur Radiation, die bis jetzt in so ungenauer und falscher Weise begrifflich fixiert und von einigen als eine Ausströmung von geistigen Spezies und von anderen als ein in Bewègunggésetztwerden des Mediums, das sukzessiverweise bis zum Auge sich fortpflanzt, bezeichnet wurde und anderseits zum Sinnesakte, wodúrch ich ebenfalls das der Öffentlichkeit preisgäbe, was ich verbergen will, so will ich das auslassen. Auch bestreite ich nicht, daß jene Bewegung, dié ich ,freeing 
of a direction" nenne, in den bestehenden Philosophien (soweit eben so eine auf schwachen Füßen stehende Antizipation festen Halt fassen. konnte) aufgedeckt und nachgewiesen werden könnte; da sie nicht viel anderes ist als das, wonach man in den zwei obengenannten Axiomen hinstrebte, aber noch mehr das, was man die Form oder die formale Ursache genannt hat oder die differentia vera; beides scheint man aber dennoch mehr als Unmöglichkeiten und Wünsche als als Dinge zu halten, die im Bereiche der menschlichen Fassungskraft liegen. Denn Plato sagt, daß er den als einen Gott anbeten würde, der der Wahrheit gemäß definieren könnte; das kann aber nur mittels der Angabe der wahren Formen und Differenzen erfolgen. Darin bin ich mit ihm einverstanden und bekenne für mich nur weniges in Anspruch nehmen zu wollen; denn wenn irgend jemand -kraft seiner Antizipationen allein die Formen entdecken kann, so werde ich ihn am meisten ehren. Da aber manche sagen würden, daß sie, wenn einige Dinge, die viele Menschen durch Unterricht und Beobachtung kennen; jemand durch Offenbarung und ohne jene Mittel erkennen könnte; sie ihn für etwas Übernatürliches und Göttliches halten würden; so bekenne auch ich, daß, wenn irgend jemand durch Antizipationen das erreichen könnte; was ein schwacher und minderwertiger Geist allein durch Interpretation erreichen kann, er keinen genug hohen Namen erhalten kann. Ja, meistenteils bin ich voll Bewunderung, zu sehen, wie weit einige unter ihnen durch bloße Antizipationen gelangt sind; aber istes nicht ebenso, als ob ich mich über die Blinden wundern würde, wenn ich sehe, welche Kunstgriffe sie ohne zu sehen machen, indem ich denke, daß ich, falls ich blind wäre, es so zu tun kaum im stande wäre. Des Aristoteles Schule behauptet, es gäbe keine wahre Erkenntnis, als bloß mittels der Ursachen, es gäbe keine wahre Ursache, als bloß die Form, eine einzige wahre Form sei bekannt - das geben sie zu; und deshalb geben sie insoweit ein für unsere Lehre günstiges Zeugnis ab, daß nämlich erstens es bis jetzt nur èinen Schatten von Erkenntnis gegeben hat und zweitens, daß wir jetzt das vorschlagen, was nach allgemeiner Zustimmung das am meisten des Aufgesuchtwerden Wärdige, aber am schwersten Auffindbare ist... Es ist da noch ein sehr notwendiger Teil nötig, nicht als Zugabe, sondern als Vorsichtsmaßregel; denn da man fast überall beobachten kann, daß die äußeren Zeichen und Merkmale der Vortrefflichkeit und Vollkommenheit mehr jenen Dingen anhaften, die blob verfälscht sind, als jenen, die 
wahr, aber von geringerer und gemeinerer Art sind, wie z. B. ein Dublin einem vollkommenen Rubin ähnlicher ist als ein Spinell umd ein verfälschter Engelstaler einem wahren Engelstaler ähnlicher sielht, als wenn es sich um einen aus Chinagold geschlagenen Engelstaller handelte; in ähnlicher Weise trägt manche „Richtung" den Schein einer in „Wahrheit" und „Freiheit" echten ,Richtung", die überhaupt keine „Richtung" ist, an sich. Denn obwohl eine „Richtung" gewib und frei zu sein scheint, indem sie uns auf eine.Natur weist, die von der von uns in Untersuchung gezogenen Natur untrennbar ist, so ist sie dennoch nur oberflächlich und verfälscht, wenn sie uns nicht um eine Stufe näher an die Handlung oder an das Licht, auf Grund dessen wir etwas tun oder erzeugen können, bringt. Deshalb will ich, um fest zu bestimmen und sicherzustellen, was eine wahre „Richtung“ ist - obwohl die allgemeine Beschreibung, welche ich gegeben habe, in sich selbst klar ist, denn jedermann wird bald mit sich selbst darüber im Klaren sein, ob er der Wirkung und Handlung näher ist, oder ob er nur einen abstrakten und verschwommenen Begriff gewonnen hat zur besseren Belehrung drei besondere Arten der Vorsicht angeben. Die erste lautet folgendermaßen: die entdeckte Natur muß ursprünglicher als die untersuchte und nicht sekundärer sein oder demselben Grade angehören, dem die das Objekt der Untersuchung bildende Natur angehört. Wie zum Beispiel: handelt es sich darum, einen Stein glänzend oder glatt zu machen, so ist es eine echte "Richtung" zu sagen: mache ihn gleichmäßig; aber handelt es sich darum, einen Stein gleichmäßig zu machen, so ist es keine gute „Richtung“" zu sagen: mache ihn glänzend oder mache ihn glatt; denn es besteht das Naturgesetz, daß die Disposition eines Dinges in bezug auf seinen eigenen Zustand oder auf seine Teile ursprünglicher ist als das, was in einer Beziehung oder in einem Übergehen zu einem anderen Dinge-besteht. So ist die Gleichmäßigkeit die Disposition des Steines in sich selbst, aber glatt ist ein Stein nur in bezug auf die Hand, die ihn antastet, und glänzend ist er bloß im Verhältnis auf das Auge, das ihn anschaut, und dennoch sind alle diese Eigenschaften des Steines zu einem Bündel zusammen verwachsen. Die ,Richtung“ ist aber unvollkommener, wenn sie uns auf eine solche nur in einer Beziehung bestehende Eigenschaft hinweist, die in eben demselben Grade eine Beziehungs-Eigenschaft wie die untersuchte Natur ist und nicht in einem von ihr verschiedenen Grade. Denn in der „Richtung“: Glanz durch Glätte zu 
errzeugen, kann man durch die Angabe dieser „Richtung“, obwohl man durch dieselbe eigentlich keine weitere, der Handlung nähere Sstufe erreicht und sie uns niemals neue, früher unbekannte Tatsachen zur Kenntnis bringt, auf dem Wege der Suggestion oder der Weckung dler Aufmerksamkeit auf einzelne Tatsachen gebracht werden, die man zwar kannte, sich aber auf dieselben gerade nicht entsann; wie man sieh ja leichter auf praktische Mittel, die Glattheit zu erzeugen, erimnert, als wenn man seine Aufmerksamkeit nur auf den Glanz gerichtet hält. Aber wenn die „Richtung" um Glanz durch Spiegelung hervor\#ubringen folgendermaßen lautete: mache die Spiegelung so, daß du dlein Antlitz darin sehen kannst, so ist eine solche „Richtung" bloß sekundär und gewährt uns keine Hilfe, denn weder bringt sie uns eine meue Kenntnis, noch suggeriert sie uns etwas. So zum Beispiel, wenn man bei der Untersuchung der Weiße gewiesen würde, eine solche Farbe zu erzeugen, die am weitesten im Dunkeln gesehen werden kann, so hätte man in einem solchen Falle ganz und gar keinen Fortschritt gemacht. Denn solche Arten von Naturen sind bloße Eigenschaften, Wirkungen, Umstände, Zufälligkeiten oder wie man sie auch benennen möge; und nicht wurzelhafte und die untersuchte Natur formierende Naturen. Die zweite Vorsichtsmaßregel besteht darin, daß die untersuchte Natur durch Trennung vor der Zusammensetzung zu einem Begriffe gebildet werde oder um genauer zu reden, durch relative Zusammenstellung bevor man zur absoluten schreitet und so weiter.

\section{Das Ziel der Methode. Erkennen und Handeln.}

Im ersten Kapitel des Valerius Terminus; das of the limits and end of knowledge betitelt ist, gibt uns Bacon eine auf den ersten Blick wohl merkwürdig anmutende Einführung in sein Werk: Es ist da viel von Gott die Rede, es werden mehrere Bibelstellen zitiert und man könnte wirklich der Meinung sein, daß er es hier nur mit einem Thema zu tun hat, das namentlich zu seiner Zeit die Geister viel beschäftigte; nämlich die Beziehungen zu besprechen, die zwischen Religion oder Theologie und Philosophie, namentlich aber Naturphilosophie herüberund hinüberlaufen. In der Tat stellt Bacon fest - wie es auch aus dem N. 0 . sattsam bekannt ist - daß nur die pseudowissenschaftlichen Bestrebungen des Menschen auf dem Gebiete der Naturphilosophie und Naturwissenschaft ihn von der Religion und dem Glauben ab- 
bringen können, während wahre Naturerkenntnis zu Gott, nämlich zu seinem Wirken in der Natur und dureh die Natur, hinführt. Eine oberflächliche Beschäftigung mit der Naturphilosophie kann den Menschen zu einem Atheisten machen, aber ein tieferes Eindringen in das Studium der Natur bringt ibn zurück zur Religion ${ }^{10}$ ). Doch ist es Bacon nicht hauptsächlich darum zu tun. Auch der Beweis mittels Bibelzitate - daß die Religion die Naturwissenschaft zuläBt, ja das Studium derselben sogar empfiehlt, ist nicht der Brennpunkt des Kapitels. Es handelt sich um etwas anderes, das in Bacons Augen eine ganz außerordentliche Bedeutung haben mußte. Seine Grundintuition, die Basis seines ganzen Denkens, nämlich den Vorzug des Handelns vor dem Denken, der Praxis vor der Theorie, sucht er r eligiös zu begründen. Wir hätten besser the ologisch sagen sollen, das heißt mit Argumenten, die in der Theologie gebraucht werden können, denn wir glauben nicht, daß Bacon auch tief religiös empfinden konnte. Es muß wieder einmal die Bibel herhalten und sich eine gewalttätige Interpretation gefallen lassen. Daß Bacon zur Bibel greift, darf uns nicht in Erstaunen setzen; er war ja ein Engländer und wird, wie es sich für einen Engländer der damaligen Zeit paßte, bibelfest genug gewesen sein - seine Mutter gehörte, wenn wir uns nicht irren, einer streng puritanischen Familie an - andererseits war es gerade ein Hang jener Zeit, die Bibel als Zeuge für alles mögliche anzurufen, so daß noch Spinoza es im ,Theologischen-politischen Traktat" nicht verschmäht hat, den Rationalismus in die Bibel hinein zu interpretieren und König Salomon zum Prototypen des rationalistisehen Denkers zu stempeln ${ }^{11}$ ): Die Erkenntnis, sagt Bacon, ist ein gefährliches Ding. Sie kann leicht zur Überhebung und zum Unglück des Menschen, führen. Anders verhält es sich mit der Giite oder Liebe: Diese hat noch keinen Menschen oder Geist zu Fall gebracht ${ }^{12}$ ). Der Mensch kann

10) V. T. ch. I (III. 221): And most sure it is, and a true conclusion of experience, that a little natural philosophy inclineth the mind to atheism, but a further proceeding bringeth the mind back to religion.

11) Tract. Theolog. - polit. cap. II., S. 382, Bd. I. der Ausgabe Van Vloten-Land (Haag 1895).

12) V. T. cap. 1. (III. 217): but in pursuit towards the similitude of God's goodness or lcve (which is one thing, for love is ncthing else but goodness put in motion or applied) neither man or spirit ever hath transgressed, or shall transgress. Die Engel find gefallen, weil sie within the oracle of knowledge tommen wollten; ebenso der.Mensch. S. 217/218: But as to the gocdness of 
( es Gott in der Erkenntnis nicht gleich machen ${ }^{13}$ ), er kann nicht in ( der Erkenntnis Gott ähnlich werden, sondern nur in der Liebe. Aber was ist die Liebe? Die Liebe ist die zum Wohle der Mensch. heit ausgeführte Handlung. Je mehr wir also unsere Handlungén dem allgemeinen Besten widmen, je mehr wir handeln, desto ähnlicher werden wir Gott. Macht und Kenntnisse, die nicht auf die Handlung abzielen, sind wertlos. The be nefit and relief of the state and society of man, das muß das Ziel unseres Strebens sein. And again - sagt Bacon - the same author doth notably disavow, both power and knowledge such as is not dedicated to goodness or love, for saith he, If I have all faith so as I could remove mountains, (there is power active) if I render my body to the fire (there is power passive), if I speak with the tongues of men and angels (there is knowledge, for language is but the conveyance of knowledge) all were nothing:4). Deshalb ist es nicht Ehre, Ruhm, Wißbegierde oder ganz praktische ${ }^{1}$.) und beschränkte Ziele, die der Mensch seinen Kenntnissen setzen soll, sondern es handelt sich darum, die Kenntnisse der Wiedereinsetzung und Wiederherstellung des. Menschen in jene Macht und Herrschaft; die er gleich nach der Erschaffung und vor dem Sündenfalle über alles Erschaffene innehatte zu widmen ${ }^{16}$ ). Diese Macht besteht aber darin, Neues zu entdecken und erfinden zu können, von der Unsterblichkeit an bis zu den geringfügigsten mechanischen Fertigkeiten ${ }^{17}$ ). Also

God, there is no danger in contending or advancing towards a similitude thereof, as that which is open and propunded to our imitation.

13) Auch kann der Mensch Gott unmittelbar nicht erkennen. S. 218: And this appeareth sufficiently in that there is no prcceeding in invention of knowledge but by similitude; and God is only self-like, having nothing in common with any creature, otherwise than as in shadow and trope.

14) V. T. ch. 1 (III. 222).

15) Lucre of profession (III. 222), das heißt der Vorteil, den man aus seinen Kenntnissen für den eigenen Beruf erzielen kann. Zielt unsere Handlung auf ein solches Ziel ab, so ist unsere Kenntnis in den Dienst einer schlechten Sache gestellt, unsere Kenntnisse dienen utilistischen Zwecken. Nur solche Zwecke sind nach Bacon also utilistisch zu nennen. Er kam sich also ganz und gar nicht als Utilist vor.

16) 1. c.: but it is a restitution and reinvesting (in great part) of man to the sovereignty and power (for whensoever he shall be able to call the creatures by their true names he shall again command them) [Anspielung auf Gen.2, und auf seine eigene Lehre] which he had in his first state of creation.

17) 1. c. And to speak plainly and clearly, it is a discevery of all operations 
auch Alchemie und Magie gehören zu den höchsten Leistungen des Menschen, denn sie gehen ja auf die Erfindung und Entdeckung neuer Mittel und Wege aus, um die Macht des Menschen über die Natur zu erhöhen. In der Tat ist die ganze baconische Philosophie nichts anderes als eine streng wissenschaftiche und ernst aufgefabte Anleitung, vorderhand wenigstens. um Naturen zu erzeugen, um dann aber auch die Hervorbringung derSubstanzen, welche eben aus Naturen zusammengesetzt sind, in Angriff nehmen zu können. Wir sehen also, daß schon in den allerfrühesten Perioden der bac nischen Spekulation der Gedanke, daß die Handlung, das Hervorbringen, das Erzeugen, die Aktion oder Operation, vor der Erkenntnis de!! Vorzug verdient, eine bedeutende Rolle spielte. Denn nicht umson $s i$ und einer geringfügigen Sache willen hätte er zur Bibel gegriffen. Er will einen Wall aufrichten, damit die Wasser der Erkenntnis nichi zu mächtig anschwellen: jede Kenntnis wird durch die Religion eingeschränkt und muß auf das Handeln gerichtet werden ${ }^{18}$ ). Den ersten Teil dieses Satzes können wir ruhig übergehen, er spielt bei Bacon keine Rolle; der zweite ist seine Grundintuition.

Wir kennen nun die geistige Atmosphäre, in der das Denken unseres Philosophen sich bewegen wird. Das 11. Kapitel des V. T. ist nichts anderes als die wissenschaftliche Begründung, des Weges, den der Mensch zubeschreiten hat, um sicher und ohne Fehlern ausgesetztzu sein, seine, ,Handlungen", actions und operations ausführen zu können. Eine solche Begründung und Anleitung ist die erste Form, in der sich uns bei Bacon der Begrift der Methode darbietet. Diese hat also nicht den Menschen zur Erwerbung von Kenntnissen anzuleiten, sondern zur Ausführung yon „Handlungen“.

Das Ziel, auf das die baconische Methode nach seiner Darlegung derselben im vorliegenden Kapitel des V. T. zusteuert, ist also $\mathrm{nicht}$

and possibilities of operations from immortality (if it were possible) to the meanest mechanical practice.

18) V. T. ch. 1. (III. 218): I thought it good and necessary in the first place to make a strong and sound head or bank to rule and guide the course of the waters; by setting down this position or firmament, namely, that all knowledge is to be limited by religion, and to be referred to use and action. 
Erkenntnis, sondern Handlung. Das heißt soviel, als daß seine Methode uns in erster Linie nicht neue Kenntnisse verschaffen, ssondern uns dazu befähigen soll, neue Entdeckungen zu machen und uns neue Handlungsweisen zu verschaffen. Um für diese Tendenz 'des baconischen Denkens ein Wort zu gebrauchen, das in Kürze uns sschon durch seine Grundbedeutung die Ansicht Bacons wiederspiegele, werden wir diese Tendenz, die wir eben kurz skizziert haben, als eine pragmat is t ische, auf die $\pi \rho \tilde{\alpha} \xi \iota s$, nicht auf die $\vartheta \varepsilon \omega \rho i ́ a$ gerichtete Tbezeichnen, wobei wir aber gleich jetzt, um möglichen Mißverständnissen vorzubeugen, erwähnen wollen, daß wir auf streng philosophiegeschichtlichem Boden stehen und folglich wir uns ganz und gar der Aufgabe als überhoben betrachten, zu untersuchen, ob Bacons Pragmatismus etwa mit der modernen philosophischen Richtung gleichen Namens übereinstimmt oder nicht. Zwar dürfte der baconische Pragmatismus sich mit dem modernen, logisch betrachtet, decken auch Bacon sagt ja schließlich: wahr ist, was sich praktisch bewährt -, aber wenn wir etwas weiter in Bacons pragmatistische Denkrichtung eindringen, so werden wir anderseits auch der großen Unterschiede gewahr, die diese beiden Typen des Pragmatismus von einander scheiden. Es ist nicht Aufgabe des Historikers der Philosophie, die Unterschiede zwischen verschiedenen Denkern oder verschiedenen Typen einer und derselben Denkrichtung zu verwischen, sondern im Gegenteil die Besonderheiten, das Nie-mehr-wiederkehrende jedes einzelnen Falles praktisch und wie es sich in der realen Konkretheit gibt, hervorzuheben. Wenn wir sagen, daß die $\pi \rho \tilde{\alpha} \xi \iota \varsigma$ das Ziel der Methode Bacons ist, so wollen wir dieses Wort in philosophischerem Sinne gemeint haben, als es gewöhnlich aufgefaßt wird. Die baconische Praxis deckt sich nicht mit der Technik, wie einige Forscher (Heußler, Cassirer) angenommen haben; denn Bacon zählt auch die Wissenschaft - als einen Ausschnitt aus dem Leben, - die Induktionsmethode in ganz vorzüglichem Maße zur.Praxis - und in dieser Beziehung müssen wir ihm vollkommen beitreten; - anderseits hat er aber den philosophischen Begriff der $\pi \varrho \tilde{\alpha} \xi \iota$ im Gegensatz zur $\vartheta \varepsilon \dot{\varepsilon} \varrho i \alpha$ nicht immer konsequent mit sich selbst festgehalten, sondern schwenkt doch manchmal wiederum zum Begriff der $\pi$ oinoıs (Technik) über, unterscheidet aber selbst wiederum seine $\pi \varrho \tilde{\alpha} \xi \iota s$ (actio, operatio) von der bloßen Technik, der ars, für die er gewiß keine Vorliebe bekundet. Der Begriff actio, operatio ist also ein Proteus in Bacons Händen. 
Er war sich selbst über das, was er mit den Ausdrücken agere umd operari meinte, nicht ganz klar. Was für verschiedene, weitverzweigtte Gedankengänge in diesen Worten zu einem Begriffsknäuel sich verschürzt haben, können wir ungefähr feststellen: Gedankengänge dier Alchimie und der Magie waren es, die Bacon da heraushebt und in eime wissenschaftliche Sphäre erhebt, sie mit gewissen logischen Lehren eines damals weltbekannten philosophischen Marktschreiers verbindet und daraus, aus allen diesen negativen Größen, eine Gedankeinrichtung zur Auslösung zu bringen half, die einen positiven Wert in der Geschichte des menschlichen Geistes hat. Unser Gebrauch dies Wortes Praxis ist also ein sehr weiter, der nur in ganz im allgemeinen das, was Bacon meinte, bezeichnet.

Auch sein Pragmatismus bedeutet deshalb Verschiedenes. Wir kennen dréi verschiedene Bedeutungen dieser Gedankenrichtung bei ihm unterscheiden, drei Gesichter, die der Gedanke des Vorzuges des Handelns vor dem Erkennen zeigt. Auch bei Bacon gibt es, wie bei dem modernen Pragmatisten, einen groben und einen abgeklärten Pragmatismus.

Erstens fordert er sowohl von der Wissenschaft im allgemeinen, als auch von der Philosophie, daß sie nur zu dem Zwecke getrieben werden sollen, dem Menschen die Herrschaft über die Natur zu geben, das heißt, alle möglichen Mittel, um sein geistiges und leibliches Wohl zu fördern, das imperium hominis über die Natur. Philosophie und Wissenschaft sollen die Kultur - dieses Wort im weitesten Sinne genommen fördern. Eine Philosophie, die darauf nicht bedacht ist, sondern nur des reinen Wissens halber betrieben wird; hat keinen Sinn und alle die darauf verwendete Mühe und Zeit ist vergeudet. Das ist der Standpunkt des reinen Utilismus. Doch trägt er diese Lehre mit einem gewissen. Vorbehalte auf, der aber unserer Ansicht nach dies möge gegen Jürgen Bona Meyer gesagt $\operatorname{sein}^{19}$ ) - nicht im stande

19) Jürgen Bona Meyer sucht Bacon vom Utilismus, den man ihm vorgeworfen hatte, dadurch reinzuwaschen, daß er die Forderung unseres Denkers Vorteile nur für die ganze Menschheit zu suchen, hervorhebt und sein Bestreben, die Praxis der Theorie vorzuziehen, dadurch abschwächt, daß er auf die Stellen hinweist, in denen Bacon die experimenta lucifera den experimenta fructifera vorzieht, also doch schließlich der Thecrie den höheren Rang anweist. Was den ersten Punkt betrifft, so können wir auf denselben nicht weiter eingehen, da man zur Entscheidung einer solchen Frage sich notwendigerweise auf Vor- 
ist, derselben den Charakter des Utilismus zu benehmen. Nicht dem einzelnen Menschen allein sollen die Wohltaten der wissenschaftlichen Forschung zu gute kommen, sondern der ganzen Menschheit, Zu verurteilen wäre das Beginnen desjenigen, der nur auf seinen eigenen Vorteil bedacht wäre und seinen Mitmenschen seine Erfindungen und

aussetzungen stützen muf, die unser moralisches Gewissen uns diktiert, also außerhalb einer jeden logischen Kontrolle liegen, und die mit Begriffen operieren müssen, die noch viel zu dunkel sind, als daß eine fruchtbringende Diskussion darüber stattfinden könnte. Was aber den zweiten Punkt betrifft, so ist dazu zu bemerken, daß Bacons Utilismus ein sehr raffinierter ist. Die experimenta lucifera sind wertvoller, weil sie für die Praxis von größerem Werte sind! Sie eröffnen dem Menschen neue Wege zum Handeln; die experimenta fructifera sind nur für einen beschränkten Kreis von Handlungen wertvoll, die lucifera dagegen für einen viel größeren. Also auch hier ist eigentlich die utilistische Auffassung maßgebend. Übrigens sollte man bei solchen Diskussionen immer bedenken, daß die termini theoretisch und praktisch gewöhnlich nicht im philosc phischen Sinne - Erkenntnis und Wille - gebraucht werden. Jürgen Bona Meyer scheint da von Voraussetzungen auszugehen, die jeder philosophischen Grundlage entbehren. Er scheint die Praxis als mit der Theorie, Wissenschaft für inkcmpatibel zu halten oder wenigstens als etwas, das der Wissenschaft gewissermaßen einen Makel aufdrücken könnte. Nun gibt es aber gar nicht eine „reine“, praxisfreie Wissenschaft. Der theoretische Akt des Menschengeistes, das ist der Erkenntnisakt, macht in seiner Isoliertheit noch nicht eine Wissenschaft aus; auch nicht eine einfache Summe, bei der die Relaticn zwischen ihren einzelnen Gliedern durch ein Pluszeichen ausgedrückt wird, solcher Erkenntnisakte macht die Wissenschaft aus, sondern erst ein System von Erkenntnisakten. Das ist eine Reihe von solchen Erkenntnisakten, die wir mit einander in eine logisch-methodische Beziehung und zu einem hierarchisch gegliederten Ganzen gebracht haben. In Beziehung bringen erfordert aber einen praktischen Akt unsererseits, eine Willensbewegung, eine Tätigkeit, also Praxis. Erst durch die Praxis kommt die Wissenschaft zu stande. Anders gesagt: es gibt rein praktische. und rein theoretische geistige Akte. Aber alle die vulgär und populär sogenannten „theoretischen“ Gebilde des Menschen (z. B. Wissenschaft, Philosophie usw.), ebenso wie die "praktischen“ sind in der Tat ein Gewebe von theoretischen und praktischen Akten (Erkenntnis und Wille). Das vulgäre Bewußtsein nennt ,,theoretisch" solche Gebilde, deren Zweck das Erkennen ist, , praktisch“" solche, deren Zweck das Handeln ist, wobei ausdrücklich hervorzuheben ist, daß das Einteilungskriterium der Praxis entnommen ist (Zwecksetzung). Eine solche Einteilung ist aber philosophisch unhaltbar. Solche Erwägungen müßte man sich überhaupt vor Augen halten, bevor man an ähnliche Fragen über Utilismus oder Nichtutilismus herantritt. Noch eines. Jede menschliche.Tätigkeit, also auch die Wissenschaft, ist utilistisch, denn jede menschliche Tätigkeit dient der Bedürfnisbefriedigung. 
Entdeckungen etwa vorenthielte. Das ist die e in e Seite des baconischen Pragmatismus. Belegstellen dafür anführen, hieße Eulen nach Athen tragen. Bacon sagt da nichts für seine Zeit Neues. Denn es war eben der Geist jener Zeit aufs Handeln, auf das Praktische gerichtet.

Einen stärkeren, feineren aber auch bedenklicheren Pragmatismus schlägt er ein, wenn er Lehrsätze und Urteile für wertlos und falsch erachtet, die nicht in den Dienst der Praxis gestellt werden können oder wenn er die Wahrheit eines Satzes, Urteiles von dessen praktischer Betätigung und Bewährung ahhängig macht. Das klassische Beispiel für den ersteren Fall - und zugleich ein Beweis, daß Bacon es mit seinem Pragmatismus sehr ernst nahm (er war seine Grundintuition) - ist die Lehre von der causa finalis. Die Untersuchung dieser causa schließt er aus der Wissenschaft aus und läßt sie höchstens in der Metaphysik - die Metaphysik ist ein Zeitvertreib für Faulenzer - gelten. Die Finalursache hat keinen Platz in der Wissenschaft, ist also vom wissenschaftlichen Standpunkte aus zu verwerfen - we il wir durch dieUntersuchung undErfassung derselben nicht zu neuen Handlungen gefüht werden. Sie ist unfruchtbar, non parit opera, unpraktisch, deshalb falsch! Der Schritt zur weiteren Behauptung, daß es eine solche gar nicht in der Natur gibt, ist sehr nahe. Bacon identifiziert nämlich - wie wir noch bei der näheren Besprechung des V. T. sehen werden - das Mittel, das zur Hervorbringung irgend eines Dinges, Vorganges oder Phänomens nötig ist, mit der causa formalis dieses Vorganges, Dinges usw., die denselben in der Natur hervorbringt. Wir bamerken nur ganz kurz, daß die causa formalis Bacons mit der aristotelisch-scholastischen causa gleichen Namens n i c ht identisch ist. Doch darüber noch später. Kennen wir nun diese causa formalis oder forma schlechtweg, aus der der Vorgang gleichsam hervorquillt (fontes rerum) und ist sie $\mathrm{zu}$ gleicher Zeit in unserem Machtbereich, so können wir jenen Vorgang oder jenes Phänomen hervorbringen. Die Kenntnis der forma ist also zur praktischen Betätigung unerläßlich. Was können wir dagegen mit der causa finalis anfangen? Das ist mit dem Zielpunkte, auf das ein Vorgang oder èin Phänomen gerichtet ist? Nimmer werden wir imstande sein, diesen Vorgang damit zu erzeugen! Deshalb weg mit der causa finalis, sie ist falsch, zum mindesten keine gute, brauchbare Arbeitshypothese (wir meinen die wissenschaftliche Arbeit). Höchstens in der Metaphysik - der reinen Theorie 
- mag sie ihr Dasein fristen. Bacon erkennt also die Existenz einer causa finalis an, doch damit soll sich nicht die Wissenschaft, die Physik beschäftigen. Da er aber von Metaphysik und Physik doch die Physik für das Wertvollere und Ausschlaggebende hält, so ist im Grunde genommen seine Lehre über die causa finalis einer Leugnung der Existenz einer Zweckursache gleich zu achten. Das wäre ein Beispiel für die zweite Art seines Pragmatismus. Daß er das Kriterium für die Wahrheit oder Falschheit eines Urteiles in der praktischen Bestätigung und Bewährung seines Inhaltes sieht, wird später noch zur Sprache kommen. Freilich ist die Anwendung eines solchen Kriteriums nur in sehr beschränktem Maße möglich. Denn es gibt eine ganze Menge von Urteilen, deren Inhalt sich nie praktisch bewähren oder bestätigen kann. Doch gebraucht Bacon dieses Kriterium der Wahrheit nur fük eine bestimmte Art von Urteilen, die er „Richtungen“ nennt und die physische Tatbestände, genauer die Formalursache eines Vorganges oder Phänomens angeben und.die ihre praktische $\mathrm{Be}$ stätigung in der Hervorbringung dieser Vorgänge seitens des Menschen finden sollen.

Einer dritten Art von Pragmatismus huldigt Bacon, wenn er Handlung und Erkenntnis, Theorie und Praxis gleichsetzt. Zu einer logisch einwandfreien Ausarbeitung dieser Lehre hat er es nicht gebracht; sie ist wohl nur als eine aphoristische, prägnante und etwas übertriebene Ausdrucksweise für seine allgemeine pragmatistische Denkrichtung zu betrachten. Doch versucht er in der Partis instaurationis secundae delineatio et argumentum ${ }^{20}$ ) klar und bündig seine Lehre zu formulieren; dabei betrachtet er das Werk, die Betätigung des Verstandes als natura unicum - also Erkenntnis und Handlung sind in ihrem innersten Wesen identisch, sie sind beide eine Betätigung des menschlichen Geistes und in eben dieser Betätigung besteht der Geist - aber fine et usu geminum. (Diese Betonung des Umstandes, daß der.Gegenstand, auf den die Handlung gerichtet ist, die Handlung kennzeichnet, ist scholastisch.) Aut enim scire et contemplari aut agere et efficere homini pro fine est. Deshalb betrachtet und erkennt der Mensch in der Wissenschaft (cognitio et contemplatio: „Theorie" iiberhaupt) die Ursache, in der Praxis sucht er die potestas et copia effecti. Worin identifizieren sich aber Theorie und Praxis? Darin,

20) Compl. works, III, $553 / 554$. 
daß dati effectus vel naturae (diese Gleichstellung muß hervorgehuben werden) quovis in subjecto causas nosse, intentio est humanae scientiae. Atque rursus, super datam materiae basin effectum quodvis sive uaturam imponere v̀el superinducere, intentio est humanae potentiae. Diese beiden Bestrebungen sind aber identisch, weil sie in idem coincidunt. Nam quod in contemplatione instar causae est, in operatione est instar medii; scimus enim per causas, operamur per media. Die Ursache (forma) eines Vorganges und dessen Hervorbringungsmittel von seiten des Menschen decken sich also. Deshalb sind nach Bacon Theorie und Praxis identisch. Diese letzten Konsequenzen seines pragmatistischen Grundgedankens sind für ihn nicht nur im V. T. von Bedeutung geworden, sondern sie sind in seinem ganzen System latent und vorausgesetzt. Sie waren sein echte's philosophisches Erlebnis. Und deshalb bilden sie den roten Faden, der Formenlehre und Methode durchzieht und sie sehr innig verbindet, ja schließlich zusammenfallen läßt. Aus demselben Grunde, aus dem Theorie und Praxis nach ihm zusammenfallen, fallen unserer Ansicht nach auch Formenlehre und Methode zusammen. Die Methode ist der Ausdruck der Praxis, die Formenlehre der der Theorie.

Die wirkliche Methode ist also die Praxis, das Handeln. Aber das Handeln ist zugleich das Ziel der Methode: Und daran haben es die früheren Philosophen gar sehr fehlén lassen und uns kein Handbuch geschenkt, welches Regeln über diesen Gegenstand enthielte ${ }^{21}$ ). Bacon dägegen tritt mit dem Anspruch auf, solche Regeln geben zu wollen. Näher bezeichnet er das, wozu uns seine Methode befähigen soll, als eine Hervorbringung von natures. Wenn er Regeln darüber aufstellen will, so meint er damit, daß die auf Grund seiner Methode vom Menschen ins Werk gesetzte Tätigkeit, eine auf ihr Ziel ohne Fehler und Abirrungen lossteuernde sein wird, wodurch sie sich eben von der ars - modern Technik oder Kunstfertigkeit --, die von Fehlern und Mutmaßungen (errors and conjectures) begleitet ist, und von dem Experimentieren (experience, an dieser Stelle soviel als aufs Geratewohl Experimentieren), welches langwierig und schwer ist, unterscheidet (2). Wichtig sind da die negativen Bestimmungen, die Bacon

21) Valerius Terminus, 11. Kap. (1). 
hier, von dem weiter unten zu erörternden „Richtungs"-begriffe gibt. Die ars fußt auf bloßen Mutmaßungen - denn so meint er es - und die auf solche Mutmaßungen fundierte Handlung kann nur Fehler im Gefolge haben, das heißt, die Handlung verfehlt ihr Ziel. Wir wollen hier nicht all das Schiefe, das in dieser Auffassung der ,Fehlerhaftigkeit" der Handlung liegt - denn eine fehlerhafte Handlung ist ein Widerspruch mit sich selbst - hervorheben, sondern nur bemerken, daß dies die zu seiner Zeit landläufige Ansicht über die Beziehung der Theorie zur Praxis ist, er also hier noch keinen Fortschritt über die vorgefundene Position trotz seines Pragmatismus macht und der Handlung gegenüber der Erkenntnis, dem Urteil, eine solche Stellung der Unterordnung und der Determiniertheit-zuweist, die ganz und gar nicht den in seinen Invektiven gegen die nur für die Theorie empfänglichen Philosophen niedergelegten Ansichten entspricht.

Anderseits ist die Handlung das Ziel der Erkenntnis (3), und viele Irrtümer sind nur dadurch entstanden, daß man dieses Ziel der Erkenntnis nicht immer im Auge hatte. Worauf es hier Bacon ankommt, geht aus diesen Worten nicht klar hervor; es läßt sich aber durch Heranziehung ähnlicher Stellen aus anderen Werken soviel erschließen, daß er auf das Kriterium der wahren Erkenntnis anspielt, welches seiner Meinung nach besagt, daß jener Ausspruch, jenes Urteil wahr ist, dessen Inhalt sich praktisch bewährt. Diese Ansicht spricht er, soweit wir sehen können, nur an einer einzigen Stelle seiner Werke aus, aber vorausgesetzt ist sie überall. Daß es ihm damit ernst war, beweist der V. T. zur Genüge. 